\title{
Vaginal Approaches Using Synthetic Mesh to Treat Pelvic Organ Prolapse
}

\author{
Jei Won Moon, Hee Dong Chae \\ Department of Obstetrics and Gynecology, Asan Medical Center, University of Ulsan College of Medicine, Seoul, Korea
}

Pelvic organ prolapse (POP) is a very common condition in elderly women. In women with POP, a sacrocolpopexy or a vaginal hysterectomy with anterior and posterior colporrhaphy has long been considered as the gold standard of treatment. However, in recent decades, the tendency to use a vaginal approach with mesh for POP surgery has been increasing. A vaginal approach using mesh has many advantages, such as its being less invasive than an abdominal approach and easier to do than a laparoscopic approach and its having a lower recurrence rate than a traditional approach. However, the advantages of a vaginal approach with mesh for POP surgery must be weighed against the disadvantages. Specific complications that have been reported when using mesh in POP procedures are mesh erosion, dyspareunia, hematomas, urinary incontinence and so on, and evidence supporting the use of transvaginal surgery with mesh is still lacking. Hence, surgeons should understand the details of the surgical pelvic anatomy, the various surgical techniques for POP surgery, including using mesh, and the possible side effects of using mesh.

Keywords: Pelvic organ prolapse; Mesh

\section{INTRODUCTION}

Pelvic organ prolapse (POP) is a very common condition seen in aged women. It was reported that "the demand for care for pelvic floor disorders would increase by 35\% between 2010 and 2030" in the United States [1]. The causes of POP are variable and mostly contribute to a weakening of the pelvic supportive structures, including connective tissues and muscles, and to damage of nerves nearby. Likewise, our society is an aging society, so the frequency of POP surgery is increasing. In the past, conventional abdominal or vaginal surgery was performed to treat patients with a POP. However, considering the patient's old age, when developing a new method of POP surgery using synthetic mesh, we should consider minimally invasive surgery, which has a lower risk than

Received: September 8, 2015 - Accepted: October 21, 2015

Correspondence to: Hee Dong Chae, M.D.

Department of Obstetrics and Gynecology, Asan Medical Center, University of Ulsan College of Medicine, 88 Olympic-ro 43-gil, Songpa-gu, Seoul 05505, Korea

Tel: +82-2-3010-3628, Fax: +82-2-3010-6944

E-mail: hdchae@amc.seoul.kr

(C) 2016 The Korean Society of Coloproctology

This is an open-access article distributed under the terms of the Creative Commons Attribution NonCommercial License (http://creativecommons.org/licenses/by-nc/4.0) which permits unrestricted noncommercial use, distribution, and reproduction in any medium, provided the original work is properly cited. conventional treatment.

The advantages of vaginal surgery using mesh for treating patients with a POP are as follows: it is less invasive than an abdominal approach, is easier to do than a laparoscopic approach, and has a lower recurrence rate than a traditional approach. However, in 2008 and 2011, the U.S. Food and drug Administration (FDA) released a statement alerting the public about the potentially serious complications and problems related to the use of transvaginal mesh in the treatment of patients with a POP. Ironically, after the 2008 FDA announcement, the rate of using vaginal-mesh implantation increased until 2009 [2]. Also, some researchers have different ways of thinking, and there is some dispute about the safety of using mesh [3]. Further research is needed to assess the longterm outcome, functional results, and the side effects of using vaginal mesh.

Until now, the vaginal approach using mesh for the treatment of patients with a POP has shown favorable results. It may decrease morbidity and preserve (or improve) surgical cure rates. The ultimate goal of POP surgery is to correct both the anatomic and the functional derangements at the same time and to improve the quality of life (QoL) of the patients, and for this purpose, the surgeon should understand in detail the surgical pelvic anatomy, the various surgical techniques including the use of mesh, and the possible side effects of using mesh. 


\section{PELVIC ANATOMY}

The pelvic anatomy may be defined as those bones, muscles, ligaments, and organs that contribute to the normal function of the pelvic floor. Normal functioning of these pelvic organs depends directly on the structural integrity of the pelvic floor. Among these, bones and connective tissue are the main structural components of the pelvis. The connective tissue consists of ligaments and fascia. The critical elements of connective tissue are collagen and elastin, both of which are changed during pregnancy, childbirth, and aging. These changes may weaken the ligaments and fascia, affecting the structural integrity of the pelvic floor. This may result in prolapse and pelvic organ dysfunction.

The axis of pelvic support was suggested by DeLancey (Fig. 1) [4, 5]. Level I is the upper vertical axis, which suspends the upper vagina and cervix from the lateral pelvic wall. Level II is the horizontal axis that leads to lateral and paravaginal support. Level III is the lower vertical axis, and it supports the lower third of the vagina, the urethra and the anal canal $[4,5]$. Three-directional muscle forces, the anterior portion of the pubococcygeus muscle, the levator plate, and the longitudinal muscle of the anus activate urethral and anal canal closure and help retain the positions of the organs. The levator plate and the longitudinal muscle of the anus open both the urethra during micturition when the pubococcygeus muscle relaxes and the anal canal during defecation when the puborectalis muscle relaxes. Especially, the middle portion of the vagina, level II by DeLancey $[4,5]$, is supported by the endopelvic fascia. It attaches the vagina laterally and more directly to the pelvic wall, and this attachment stretches the vagina transversely between the bladder and the rectum and has functional significance. The endopelvic fascia is attached laterally to the ar-

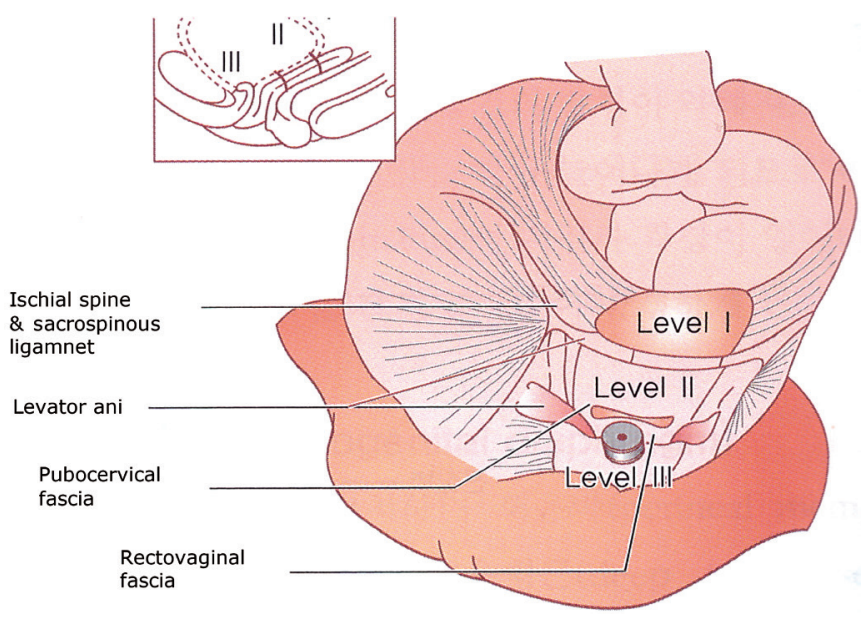

Fig. 1. Axis of pelvic support. Adapted from Korean Society of Obstetric and Gynecology, editor. Gynecology. 5th ed. 2015. Fig. 43-6; p. 1120 , with permission of Korean Society of Obstetric and Gynecology [5]. cus tendineus fascia pelvis. The arcus tendineus fascia pelvis is a thickening of the parietal fascia of the levator ani muscle and travels from the pubic arch in a straight line to the ischial spine; it is approximately $8-10 \mathrm{~cm}$ in length.

The bony pelvis is not important to the support of the pelvic organs for preventing prolapse, but several structures are very important for POP surgery: the ischial spine, the obturator foramen, the sacrospinous ligament, and the sacrotuberous ligament. The internal pudendal artery from the internal iliac artery and the pudendal nerve pass the greater and the lesser sciatic foramen around the ischial spine, and because this area is the penetration site for suspension during prolapse surgery using mesh, we should be careful during the operation (Fig. 2) [6]. Understanding the pelvic anatomy and the supporting structures as described
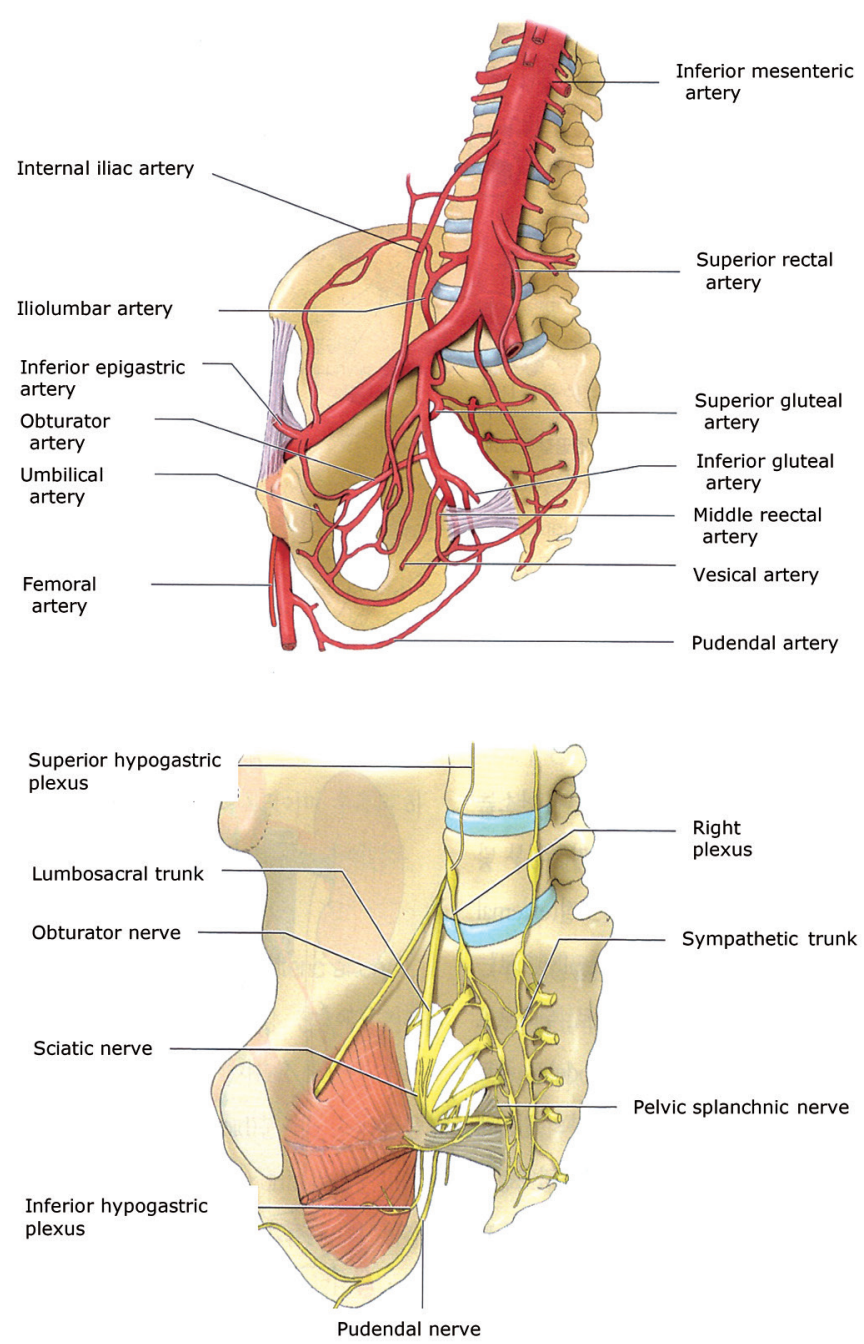

Fig. 2. Blood supply and nerve distribution in the pelvis. Adapted from Korean Society of Obstetric and Gynecology, editor. Gynecology. 5th ed. 2015. Fig. 2-3, 2-5; p. 18, 20, with permission of Korean Society of Obstetric and Gynecology [6]. 
above is very important for surgical treatment of a POP.

\section{TREATMENTS}

To treat patients with a POP, the surgeon should always consider the patient's age, the patient's desire for childbearing, the severity of the patient's symptoms, which might have an impact on the patient's QoL, the patient's desire for coital function, and the presence of other medical morbidities. After the patient's overall condition has been considered, a thoughtful decision should be made concerning the treatment of the POP, which includes nonsurgical and surgical treatments. Nonsurgical methods include lifestyle modification (weight loss and reduction of those activities that increase intra-abdominal pressure), pelvic floor muscle training such as Kegel exercise, and the use of mechanical devices. However, conservative management can only be helpful for patients with a lower POP stage according to the pelvic organ prolapse quantification system (POP-Q) [7]. The purposes of conservative treatment are to avoid or delay surgery because of old age, to reduce surgical risks, to increase muscle strength, and to prevent worsening of the symptoms.

Correcting the prolapsed vagina, while maintaining sexual function and relieving associated pelvic symptoms, is important. Many techniques have been described for POP surgeries, but unfortunately, few studies have attempted to identify the optimal surgical approach. In upper vaginal prolapse (uterine or vaginal vault prolapse), a vaginal hysterectomy with an anterior and posterior colporrhaphy, an abdominal (laparotomic, laparoscopic, or robotic) sacrocolpopexy, and a uterosacral or sacrospinous ligament suspension have been used. For an anterior or posterior vaginal wall prolapse, an anterior or posterior colporrhaphy with or without graft reinforcement should be considered.

Mesh is a metallic or polymeric screen intended to be implanted to reinforce soft tissue, fascia, or bone where weakness exists. There are synthetic and biological meshes, and biological grafts can be classified as allografts, autografts or xenografts. Synthetic meshes differ in absorbability (nonabsorbable vs. absorbable), composition (monofilament vs. multifilament), pore size, flexibility, and architecture (knitted or woven) [8]. In synthetic grafts, polypropylene mesh of a monofilament, macroporous type is most commonly used; however, the use of polypropylene mesh may cause a strong foreign body reaction, and healing may be adversely affected when such a reaction occurs. Recently, a partiallyabsorbable mesh has been used, and compared with nonabsorbable mesh, it seems to have a lower exposure rate $[9,10]$. Mesh should be sterile and noncarcinogenic, produce no inflammatory reaction, and be easy to use, nonallergenic, and resistant to infection, but unfortunately, as of now, no such ideal mesh exists.

Petros performed intravaginal sling-plasties using mesh for the first time (Fig. 3) [11]. Seventy-five women with at least a seconddegree vaginal vault prolapse after abdominal or vaginal hysterectomy were included. The success rate of that study was $94 \%$, and a

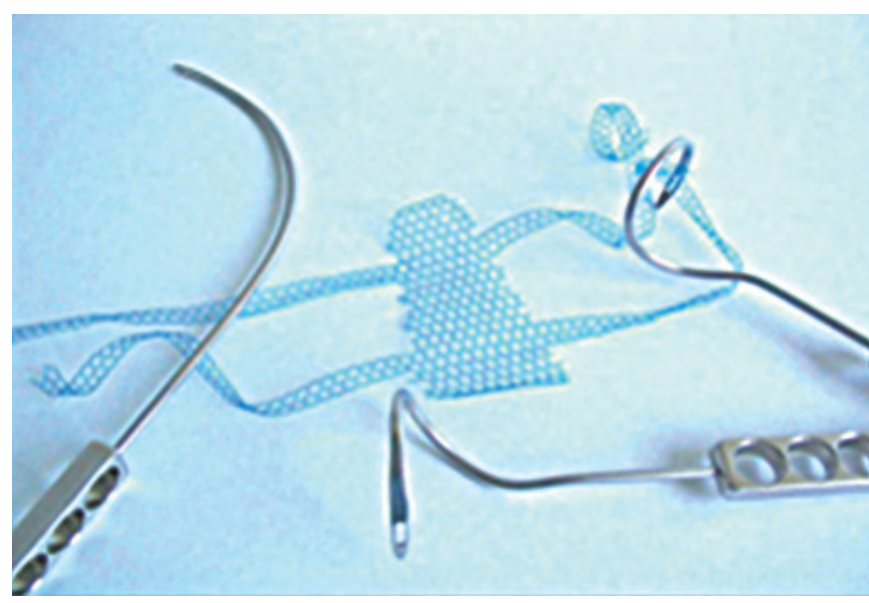

Fig. 3. Transvaginal surgical approach using mesh to treat a pelvic organ prolapse. Adapted from Serag Wiessner (Naila, Germany), with permission.

$5.6 \%$ mesh erosion rate was reported. After the advent of the total vaginal polypropylene mesh kit, POP management has experienced a complete change. Many studies have shown acceptable cure rates with low morbidity rates [12]. A recent randomized, prospective, controlled study showed that using transvaginal mesh in the treatment of a posthysterectomy vaginal vault prolapse had a lower recurrence rate than surgery with sacrospinous fixation did [13]. In that study, the sacrospinous fixation group had a higher recurrence rate ( 28 of $72,39 \%)$ than transvaginal mesh group did (13 of 79,17\%). Reoperation for prolapse was performed in 3 out of the 72 patients in the sacrospinous fixation group and in 1 out of the 79 patients in the transvaginal mesh group. No differences in the de novo stress urinary incontinence (SUI), bladder overactivity, dyspareunia, pelvic pain and QoL improvement were identified between the 2 groups. However, the mesh exposure rate has been reported to be $20.8 \%$, and several retrospective studies have shown anatomical cure rates of $81.2 \%$ $89.9 \%$ when transvaginal mesh kits are used for the treatment of patients with a POP [14]. Surprisingly, one retrospective study from Canada showed $97.4 \%$ of overall success rate, with a small mesh extrusion in only 1 patient, when light-weight polypropylene mesh was used to repair the POP [15].

Two recent randomized, controlled trial comparing traditional colporrhaphy and transvaginal polypropylene mesh for the treatment of patients with an advanced anterior vaginal prolapse showed that transvaginal polypropylene mesh surgery had higher anatomical success and satisfaction rates compared with traditional colporrhaphy [16]. A similar randomized, controlled trial concluded that trocar-guided transvaginal synthetic mesh for advanced anterior POP repair had a higher success rate than traditional colporrhaphy with a 5\% mesh erosion rate [17]. Many other studies have demonstrated that compared with native tissue repairs, transvaginal synthetic mesh surgery decreases the inci- 
dence of prolapse recurrence in the anterior compartment [18].

Although transvaginal synthetic mesh surgery has the benefits of low rates of prolapse recurrence and high satisfaction rates after the procedure, several complications are associated with transvaginal synthetic mesh surgery. In 2011, the US FDA had a consensus meeting to update the safety and the effectiveness of transvaginal placement for the treatment of patients with a POP. The most common complications of the mesh were reported to be mesh exposure, pelvic pain, infection, bleeding, dyspareunia, and organ perforation [19]. Additionally, contrary to our expectations, regarding rates of reintervention, a newly-developed partially-absorbable mesh does not seem to give any advantage in reducing mesh exposure, recurrence, urinary complication and dyspareunia in comparison with classic nonabsorbable mesh [20]. However, some risk factors for mesh erosion can be reduced. Surgical factors are improper mesh placement, inverted T-incision, vaginal-wall trimming, total application of mesh, and surgeon's experience or skill. Host factors are smoking, obesity, poorly controlled diabetes mellitus, urogenital atrophy, prior pelvic radiation, and current steroid use. Surgeons should undergo surgical training and acquire a full understanding of the role of mesh before using a vaginal approach with mesh for POP surgery. Nevertheless, vaginal mesh is an important tool in surgical armamentarium and may be the best options in some patients with a POP, especially those with an anterior compartment POP, a recurrent POP, an advanced $\mathrm{POP}$ or significant medical comorbidities in whom an invasive sacrocolpopexy procedure may be contraindicated [21].

\section{CONCLUSION}

The best approach to POP surgery remains controversial. However, a vaginal approach using mesh for the treatment of a POP may improve the surgical cure rate and decrease the burden of invasive surgery on the patient. The main purpose of using mesh is to achieve both anatomical recovery and improved QoL. When mesh is being considered for POP surgery, each patient must be carefully evaluated on an individual basis, and informed consent based on the latest information available in the literature must be obtained. The use of vaginal mesh should be limited to a carefully selected population. Surgeons should choose mesh surgery only after weighing the risks and the benefits of surgery with mesh versus all surgical and nonsurgical alternatives, notify the patient that mesh will be used in the POP surgery, and provide the patient with information on the risks associated with the use of mesh, such as complications and limited long-term outcomes.

\section{CONFLICT OF INTEREST}

No potential conflict of interest relevant to this article was reported.

\section{REFERENCES}

1. Kirby AC, Luber KM, Menefee SA. An update on the current and future demand for care of pelvic floor disorders in the United States. Am J Obstet Gynecol 2013;209:584.e1-5.

2. Reynolds WS, Gold KP, Ni S, Kaufman MR, Dmochowski RR, Penson DF. Immediate effects of the initial FDA notification on the use of surgical mesh for pelvic organ prolapse surgery in medicare beneficiaries. Neurourol Urodyn 2013;32:330-5.

3. Murphy M, Holzberg A, van Raalte H, Kohli N, Goldman HB, Lucente $\mathrm{V}$, et al. Time to rethink: an evidence-based response from pelvic surgeons to the FDA Safety Communication: "UPDATE on Serious Complications Associated with Transvaginal Placement of Surgical Mesh for Pelvic Organ Prolapse". Int Urogynecol J 2012;23: 5-9.

4. DeLancey JO. Anatomic aspects of vaginal eversion after hysterectomy. Am J Obstet Gynecol 1992;166(6 Pt 1):1717-24.

5. Korean Society of Obstetric and Gynecology, editor. Gynecology. 5th ed. Seoul: Korea Medical Book; 2015. Fig. 43-6, Structures of vaginal support; p. 1120.

6. Korean Society of Obstetric and Gynecology, editor. Gynecology. 5th ed. Seoul: Korea Medical Book; 2015. Fig. 2-3, Vessels in pelvis, 2-5, Nerves in pelvis; p. 18, 20.

7. Bump RC, Mattiasson A, Bo K, Brubaker LP, DeLancey JO, Klarskov $\mathrm{P}$, et al. The standardization of terminology of female pelvic organ prolapse and pelvic floor dysfunction. Am J Obstet Gynecol 1996;175:10-7.

8. Huebner M, Hsu Y, Fenner DE. The use of graft materials in vaginal pelvic floor surgery. Int J Gynaecol Obstet 2006;92:279-88.

9. Farthmann J, Watermann D, Niesel A, Fünfgeld C, Kraus A, Lenz F, et al. Lower exposure rates of partially absorbable mesh compared to nonabsorbable mesh for cystocele treatment: 3-year follow-up of a prospective randomized trial. Int Urogynecol J 2013;24:74958.

10. Milani AL, Hinoul P, Gauld JM, Sikirica V, van Drie D, Cosson M, et al. Trocar-guided mesh repair of vaginal prolapse using partially absorbable mesh: 1 year outcomes. Am J Obstet Gynecol 2011; 204:74.e1-8.

11. Petros PE. Vault prolapse II: restoration of dynamic vaginal supports by infracoccygeal sacropexy, an axial day-case vaginal procedure. Int Urogynecol J Pelvic Floor Dysfunct 2001;12:296-303.

12. Abdel-Fattah M, Ramsay I; West of Scotland Study Group. Retrospective multicentre study of the new minimally invasive mesh repair devices for pelvic organ prolapse. BJOG 2008;115:22-30.

13. Halaska M, Maxova K, Sottner O, Svabik K, Mlcoch M, Kolarik D, et al. A multicenter, randomized, prospective, controlled study comparing sacrospinous fixation and transvaginal mesh in the treatment of posthysterectomy vaginal vault prolapse. Am J Obstet Gynecol 2012;207:301.e1-7.

14. Khan ZA, Thomas L, Emery SJ. Outcomes and complications of trans-vaginal mesh repair using the Prolift ${ }^{\mathrm{m}}$ kit for pelvic organ prolapse at 4 years median follow-up in a tertiary referral centre. 
Arch Gynecol Obstet 2014;290:1151-7.

15. Stanford EJ, Mattox TF, Pugh CJ. Outcomes and complications of transvaginal and abdominal custom-shaped light-weight polypropylene mesh used in repair of pelvic organ prolapse. J Minim Invasive Gynecol 2011;18:64-7.

16. Dias MM, de A Castro R, Bortolini MA, Delroy CA, Martins PC, Girao MJ, et al. Two-years results of native tissue versus vaginal mesh repair in the treatment of anterior prolapse according to different success criteria: a randomized controlled trial. Neurourol Urodyn 2015 Mar 27 [Epub]. http://dx.doi.org/10.1002/nau.22740.

17. Delroy CA, Castro Rde A, Dias MM, Feldner PC Jr, Bortolini MA, Girao MJ, et al. The use of transvaginal synthetic mesh for anterior vaginal wall prolapse repair: a randomized controlled trial. Int Urogynecol J 2013;24:1899-907.
18. Dessie SG, Hacker MR, Haviland MJ, Rosenblatt PL. Attitudes toward transvaginal mesh among patients in a urogynecology practice. Int Urogynecol J 2015;26:865-73.

19. Walter JE; Urogynaecology Committee, Lovatsis D, Walter JE, Easton W, Epp A, et al. Transvaginal mesh procedures for pelvic organ prolapse. J Obstet Gynaecol Can 2011;33:168-74.

20. Quemener J, Joutel N, Lucot JP, Giraudet G, Collinet P, Rubod C, et al. Rate of re-interventions after transvaginal pelvic organ prolapse repair using partially absorbable mesh: 20 months median follow-up outcomes. Eur J Obstet Gynecol Reprod Biol 2014;175: 194-8.

21. Iglesia CB. Synthetic vaginal mesh for pelvic organ prolapse. Curr Opin Obstet Gynecol 2011;23:362-5. 